UniversitätsSpital Zürich

Dermatologische Klinik

Direktor: Prof. Dr. med. Reinhard Dummer

Arbeit unter Leitung von Prof. Dr. med. Reinhard Dummer und. Dr. med. Simone M. Goldinger

\title{
Noncutaneous Melanomas: A Single-Center Analysis
}

\author{
INAUGURAL-DISSERTATION \\ zur Erlangung der Doktorwürde der Humanmedizin \\ der Medizinischen Fakultät \\ der Universität Zürich
}

vorgelegt von

Valerio Del Prete

Genehmigt auf Antrag von Prof. Dr. med. Reinhard Dummer 


\section{Publikationshinweis}

Noncutaneous Melanomas: A Single-Center Analysis

Publiziert am: 01.12.2015

Journal: Im Dermatology (Karger Publishers), No. 1 2016, Band 232, Seiten 22-29 


\title{
Dermatology
}

\section{Noncutaneous Melanomas: A Single-Center Analysis}

\author{
Valerio Del Prete Karla Chaloupka David Holzmann Daniel Fink \\ Mitchell Levesque Reinhard Dummer Simone M. Goldinger \\ Department of Dermatology, University Hospital of Zurich, Zurich, Switzerland
}

\section{Key Words}

Mucosal melanoma - Uveal melanoma - Vulvovaginal melanoma Sinonasal melanoma $\cdot$ Ocular melanoma Noncutaneous melanoma mor-signaling pathway mutations and distinct metastasization patterns. Immunotherapy induces response rates in mucosal melanoma similar to those in cutaneous melanoma.

๑ 2015 S. Karger AG, Basel

\begin{abstract}
Background: The optimal treatment algorithm for noncutaneous melanomas must yet be established. Objective: To compare systemic treatment-relevant mutational status, metastatic pattern and response to systemic treatment in noncutaneous melanoma. Methods: Retrospective singlecenter study analyzing 64 noncutaneous melanoma patients treated between January 2006 and September 2013. Results: c-KIT mutations were found exclusively in vulvovaginal melanoma (4/7). Overall status for NRAS and BRAF mutations was low ( $1 / 7$ and $0 / 21$ detected mutations, respectively). Seven out of 7 vulvovaginal and $6 / 13$ sinonasal melanomas first metastasized to lymph nodes, whereas $18 / 22$ ocular melanomas first metastasized to the liver. Response to systemic treatment in vulvovaginal melanomas was best for imatinib with a disease control rate of $3 / 3$ and overall for ipilimumab with a disease control rate of $3 / 10$. Sorafenib was associated with adverse drug reactions (6/13) and poor results. Conclusion: Noncutaneous melanomas show few tu-
\end{abstract}

\begin{tabular}{|c|c|}
\hline KARGER & $\begin{array}{l}\text { (1) 2015 S. Karger AG, Basel } \\
1018-8665 / 15 / 2321-0022 \$ 39.50 / 0\end{array}$ \\
\hline $\begin{array}{l}\text { E-Mail karger@karger.com } \\
\text { www.karger.com/drm }\end{array}$ & \\
\hline
\end{tabular}

\section{Introduction}

Noncutaneous melanomas are uncommon tumors and form a small fraction of tumors that develop from melanocytes [1]. The majority are found in the uvea, the oronasal and genital mucosa [2]. Most of them are characterized by poor prognosis, difficult local control and rare tumors with typically late diagnosis and unsatisfactory therapy, a standardized treatment algorithm for systemic disease has not been developed for most of them yet.

In recent years, molecular staging in cancer cells and detection of activating mutations in the tumor-signaling pathway have given new therapeutic target options for cutaneous melanoma. However, only few studies have reported on the mutation status and effect of the new specific therapeutic agents in noncutaneous melanoma. This analysis reports the different genetic aberrations of tumor

Prof. Dr. med. Reinhard Dummer

Department of Dermatology

University Hospital of Zurich

Gloriastrasse 31, CH-8091 Zurich (Switzerland)

E-Mail reinhard.dummer@usz.ch high incidence for nodal and distant metastasis. Being 
cells found in our collective, evaluates the distinct metastatic patterns and compares the different therapeutic approaches and outcomes.

\section{Patients and Methods}

This is a single-center retrospective data analysis of 64 noncutaneous melanoma patients approved by the local ethics committee (KEK-ZH No. 2014-0143). Patients with sinonasal, vulvovaginal or ocular melanoma treated or aftercared at the Department of Dermatology of the University Hospital of Zurich between January 1, 2006, and September 31, 2013, were analyzed. Data was collected based on the internal medical history charts system KISIM (CISTEC AG, Zurich, Switzerland). Patients were classified into 3 subgroups (sinonasal/vulvovaginal/ocular) and evaluated with descriptive statistics separately. Each group was analyzed for primary therapy, mutation status, localization of first metastasis and systemic therapy response.

The date and localization of the first distant metastasis were defined at the first scan where metastasis suspicion was reported. When multiple first metastases in different organs were detected within the same scan, all locations were listed. A CT scan after 3 months of therapy was applied to determine treatment response. Response was graded in 4 classes according to standardized RECIST criteria: complete response (CR; $-100 \%$ of tumor mass), partial response (PR; more than $-30 \%$ of tumor mass), stable disease (SD; $-30 \%$ up to $+25 \%$ of tumor mass) and progressive disease (PD; more than $+25 \%$ of tumor mass or new tumor lesions). The disease control rate is defined as the sum of CRs, PRs and SDs. Due to multiple serial therapies with different agents, the sum of used therapeutics surmounts the number of patients in tables for systemic therapy. Mutational status (BRAF, NRAS, c-KIT) was determined by PCR and assessed routinely in previously collected tissue. No additional mutation analysis was performed for the current retrospective research project.

\section{Results}

A total of 64 patients, 17 of them with sinonasal, 8 with vulvovaginal and 39 with ocular melanoma, were evaluated.

\section{Sinonasal Melanoma}

The median age of the 17 included sinonasal patients at first diagnosis was 69 years (range 44-91), and there was a minor predominance for the female gender of $10 / 17$ $(58.9 \%)$. Recurrent nasal bleeding and persistent nasal obstruction were the most common symptoms. Tumor size reached from 1 up to $7.5 \mathrm{~cm}$ of longitudinal spreading.

As primary therapy, 16 out of 17 patients had surgery. One patient did not undergo surgery due to extended spreading. Endonasal resections were primarily per-
Table 1. Mutation status listed by melanoma subgroup

\begin{tabular}{lclc}
\hline Mutation & Sample number & Mutated & $\begin{array}{l}\text { Wild } \\
\text { type }\end{array}$ \\
\hline Sinonasal & & & \\
BRAF & 10 & 0 & 10 \\
NRAS & 4 & 1 & 3 \\
c-KIT & 9 & 0 & 9 \\
\hline Vulvovaginal & & & \\
BRAF & 5 & 0 & 5 \\
NRAS & 2 & 0 & 2 \\
c-KIT & 7 & 4 & 3 \\
\hline Ocular & & & \\
Uveal & & & \\
$\quad$ BRAF & 5 & 0 & 5 \\
$\quad$ NRAS & 1 & 0 & 1 \\
C-KIT & 2 & 0 & 2 \\
Conjunctival & & 0 & 1 \\
$\quad$ BRAF & 1 & 0 & 0 \\
$\quad$ C-KIT & 0 & 0 & 1 \\
\hline
\end{tabular}

Mutation status is listed by melanoma subgroup. Molecular tumor analysis was performed for 10 of 17 sinonasal, 5 of 8 vulvovaginal and 7 of 36 ocular melanoma patients. Mutational status (BRAF, NRAS, c-KIT) was determined by PCR and assessed routinely in previously collected tissue. No additional mutation analysis was performed for the current retrospective research project.

${ }^{1}$ Two mutations involved exon 13 K642E, 1 exon 17 N822K and 1 exon 11 L576P.

formed, whereof only one resulted directly in R0 resection. With repeat surgery, 6 additional $\mathrm{R} 0$ resections were achieved. Radiotherapy with an average total of 60-70 Gy was applied in those patients where $\mathrm{R} 0$ resection was not accomplished. In 1 case only, $\mathrm{R} 0$ resection was accompanied by radiotherapy.

Local recurrence was a problem independent of the therapeutic procedure; 8 of $9(89 \%)$ patients with R1 and radiotherapy, as well as 5 of $6(83 \%)$ patients with R0 resection and no additional adjuvant radiotherapy showed local recurrence. The patient who received adjuvant radiotherapy in addition to $\mathrm{R} 0$ resection had no local recurrence. However, local tumor control did correlate linearly neither with absence of metastasis nor with increased overall survival.

Molecular tumor analysis was performed for 10 of 17 patients. None of the analyzed sinonasal tumors were BRAF (0/10) or C-KIT (0/9) mutation positive. In 1 out of 4 patients, NRAS mutation was detected (table 1). 
Table 2. Localization of first metastasis listed by melanoma subgroup

\begin{tabular}{lc}
\hline Localization & Frequency \\
\hline Sinonasal $(\mathrm{n}=13)$ & \\
$\quad$ Lymph nodes & $6(46 \%)$ \\
Lung & $5(38 \%)$ \\
Bone & $4(31 \%)$ \\
Stomach & $1(8 \%)$ \\
Omentum & $1(8 \%)$ \\
Meningeosis carcinomatosa & $1(8 \%)$ \\
Vulvovaginal $(\mathrm{n}=7)$ & \\
Lymph nodes & $7(100 \%)$ \\
Ocular $(\mathrm{n}=22)$ & \\
Liver & $18(82 \%)$ \\
Lung & $7(32 \%)$ \\
Bone & $5(23 \%)$ \\
Lymph nodes & $3(14 \%)$ \\
Abdominal & $2(9 \%)$ \\
\hline
\end{tabular}

The sum of listed metastasis localizations can surmount total patient number due to multiple simultaneous detections with the same scan. Localization of first metastasis is listed by melanoma subgroup. Frequency is listed in numbers and percentages illustrating the different metastatic pattern of the three different subgroups: sinonasal melanoma predominantly metastasize to lymph nodes and lungs, all vulvovaginal melanomas showed lymph node involvement first, whereas ocular melanoma mainly spread to the liver.

Thirteen out of 17 (67.4\%) patients developed metastasis during the observation period after an average of 14.5 months (range $0-52.6$ ). Four $(23.5 \%)$ patients already showed metastasis at the time of first diagnosis. Localization of first metastasis varied and was most frequently observed in lymph nodes (46\%), lungs (38\%) and/or bones (31\%; table 2).

Nine of the 13 metastatic patients received single or multiple systemic therapies, and 4 patients did not qualify. When progression was noticed, further therapeutics were applied. Table 3 shows the used systemic therapeutics and their responses. Ipilimumab was given to $4 \mathrm{pa}$ tients, was overall much better tolerated than sorafenib and showed 1 total clinical and radiological tumor remission in a patient with lung, liver, cecum and bone metastases. This response was stable for over 12 months and still ongoing at the end of the study period. The most of ten prescribed therapeutic agent sorafenib $(n=6)$ showed a rather poor response and was highly associated with severe adverse side effects that led to therapy interruption in 4 of 6 cases (67\%). However, response to sorafenib seemed not to be correlating with the treatment duration, as the only patient maintaining SD was able to take medication $(2 \times 400 \mathrm{mg} /$ day p.o. $)$ merely for 10 days. The combined treatment with BRAF/MEK inhibitors [selective MEK inhibitor binimetinib (MEK162) $30 \mathrm{mg}$ orally twice a day and panBRAF inhibitor RAF $26560 \mathrm{mg}$ orally once a day] was given within a clinical trial to the NRASmutated patient and led to SD after 3 months (NCT NCT01352273). Patients with sinonasal melanoma in this cohort showed a 1-year survival of $93.75 \%$ and a 2 -year survival of $75 \%$.

\section{Vulvovaginal Melanoma}

Eight patients with vulvovaginal melanoma were analyzed. The median age at first diagnosis was 62 years (range 55-75). Larger tumors caused itching or bleeding, smaller tumors were mostly diagnosed incidentally by gynecological routine control. The macroscopic diameter of 6 measured tumors reached from 1 up to $4 \mathrm{~cm}$, and thickness ranged from 1.5 to $8 \mathrm{~mm}$. Seven of 8 patients underwent surgery for total excision. In 1 case, tumor size was too large for surgery, and due to the concomitant presence of lymph node metastasis, systemic therapy was initiated. In 5 of $7(71 \%)$ cases, R0 resection was achieved at first surgery, 2 patients needed repeat surgery resulting in good local tumor control. In 5 out of 7 patients, sentinel lymph node biopsy was positive or lymph node metastases were radiologically detected at the time of surgery. In these patients, a subsequent lymph node dissection was performed. Additional adjuvant therapy was performed in 1 case only with systemic pegylated interferon for 5 years $(100 \mu \mathrm{g} /$ week s.c.).

Molecular tumor mutation analysis was performed in 7 of 8 patients. Four samples were positive for c-KIT (4/7) mutations, containing 2 exon $13 \mathrm{~K} 642 \mathrm{E}, 1$ exon 17 N822K and 1 exon 11 L576P mutations, whereas no BRAF (0/5) or NRAS (0/2) mutations were found (table 1).

In all patients who developed metastasis during the study period (7/8), local lymph nodes were first affected (table 2).

Six of $8(75 \%)$ patients developed further distant metastasis during the study period after a median period of 24.3 months (range 0-104.8), 2 of them already at the time of first diagnosis. Applied systemic therapeutics and their responses are listed in table 3. Best responses were achieved with imatinib and ipilimumab, leading to PRs in $2 / 3$ (66\%) of cases for imatinib and $1 / 3(33 \%)$ for ipilimumab. The anti-PD-1 antibody pembrolizumab (blinded 2 or $10 \mathrm{mg} /$ $\mathrm{kg}$ i.v. every 3 weeks, NCT01704287) was given only in 1 case and led to SD after 3 months. Adjuvant treatment
24

Dermatology 2016;232:22-29

DOI: $10.1159 / 000441444$
Del Prete/Chaloupka/Holzmann/Fink/ Levesque/Dummer/Goldinger 
Table 3. Systemic therapy and response listed by melanoma subgroup

\begin{tabular}{|c|c|c|c|c|}
\hline Therapeutic agent & $\begin{array}{l}\text { Total } \\
\text { number }\end{array}$ & OR or $\mathrm{CR}$ & $\mathrm{SD}$ & PD \\
\hline \multicolumn{5}{|l|}{ Sinonasal } \\
\hline Ipilimumab & 4 & 1 & 1 & 2 \\
\hline Imatinib & 1 & 0 & 0 & 1 \\
\hline Sorafenib & 6 & 0 & $1^{1}$ & $5^{1}$ \\
\hline BRAF/MEK inhibitor & 1 & 1 & 0 & 0 \\
\hline Chemotherapy & 6 & 0 & 1 & 5 \\
\hline \multicolumn{5}{|l|}{ Vulvovaginal } \\
\hline Ipilimumab & 3 & 1 & 0 & $2^{2}$ \\
\hline Pembrolizumab & 1 & 0 & 1 & 0 \\
\hline Interferon pegintron $100 \mu \mathrm{g} /$ week s.c. & 1 & 0 & 1 & 0 \\
\hline Imatinib & 3 & 2 & 1 & 0 \\
\hline Nilotinib & 1 & 0 & 0 & 1 \\
\hline Chemotherapy & 5 & 0 & 1 & 4 \\
\hline \multicolumn{5}{|l|}{ Ocular } \\
\hline Ipilimumab & 3 & 0 & 0 & 3 \\
\hline Bevacizumab + paclitaxel + carboplatin & 1 & 0 & 1 & 0 \\
\hline Sorafenib monotherapy & 4 & 0 & 1 & $3^{3}$ \\
\hline Sorafenib + paclitaxel & 2 & 0 & 2 & 0 \\
\hline Sorafenib + cisplatin & 1 & 0 & 0 & 1 \\
\hline Selumetinib & 1 & 0 & 0 & 1 \\
\hline Sonidegib (LDE225) & 1 & 0 & 0 & 1 \\
\hline Bortezomib + temozolomide & 1 & 0 & 1 & 0 \\
\hline Dacarbazine & 7 & 0 & 2 & 5 \\
\hline Vindesine monotherapy & 2 & 0 & 1 & 1 \\
\hline Vindesine + cisplatin & 2 & 1 & 0 & 1 \\
\hline Cisplatin monotherapy & 1 & 0 & 0 & 1 \\
\hline Temozolamide monotherapy & 1 & 0 & 0 & 1 \\
\hline Paclitaxel + liposomal doxorubicin & 1 & 0 & 1 & 0 \\
\hline Taurolidine & 1 & 0 & 0 & 1 \\
\hline Lomustine & 1 & 0 & 1 & 0 \\
\hline
\end{tabular}

${ }^{1}$ Four of 6 patients had to stop treatment due to severe cutaneous toxicity (palmoplantar erythrodysesthesia). ${ }^{2}$ One of 2 patients had to stop treatment due to severe adverse drug reaction (autoimmune colitis + sigma perforation, uveitis and otitis interna). ${ }^{3}$ Two of 3 patients had to stop treatment due to cutaneous toxicity (palmoplantar erythrodysesthesia).

with pegintron $100 \mu \mathrm{g} /$ week s.c. for over 5 years (including nodal relapse during that time followed by lymph node dissection) showed a favorable course in 1 case as well, maintaining the patient disease free since then.

\section{Ocular Melanoma}

Thirty-nine patients with ocular melanoma (36 uveal, 3 conjunctival) were included into the study. The median age at first diagnosis was 62 years (overall range 28-93, uveal range 28-93, conjunctival range 61-81) with slight predominance for the female gender of $23 / 39(60 \%)$. Most common symptoms were visual impairment or retinal detachment for uveal and visible exterior color change for conjunctival melanoma. However, diagnosis occurred as incidental findings during ophthalmological routine examinations as well. Primary uveal melanoma treatment included enucleation $(n=19)$ or radiotherapy ( $n=16$, mainly proton beam therapy). One patient refused therapy. Conjunctival tumors were addressed depending on size and localization. One patient with a small limbal tumor partially spreading on the cornea underwent a full nontouch conjunctival tumor excision including resection of the corneal part in toto followed by topical $0.25 \%$ mitomycin application (33\%). In the other 2 $(67 \%)$ cases, tumor extent was larger, and an exenteration had to be performed. All excisions were in sano. 
Four out of $16(25 \%)$ uveal melanoma patients who received radiotherapy as primary treatment experienced local recurrence after an average of 43 months (range 2476). In contrast, local tumor recurrence in patients who underwent enucleation was not detected. Also, none of the 3 conjunctival melanoma patients showed local tumor recurrence.

Partial molecular tumor mutation analysis was performed in 7 of 36 patients with uveal melanoma and for 1 of 3 patients with conjunctival melanoma. No mutation was found for BRAF (0/5), NRAS (0/1) or c-KIT (0/2) in uveal melanoma, and the analyzed conjunctival tumor showed no mutation for BRAF or c-KIT either, with indefinite NRAS mutation status (table 1).

In $22(56 \%)$ patients (21 uveal, 1 limbal conjunctival primary tumor), metastasis was detected after a median period of 56.9 months (range 0-198.1). Two patients (uveal) already showed metastasis at the time of first diagnosis. The most frequent localization of first distant metastasis was the liver ( $82 \%)$, followed by the lungs $(32 \%)$ and bones (23\%; table 2).

Thirteen of the 22 patients with systemic disease qualified for systemic therapy. Applied systemic therapeutics and their responses are listed in table 3. Seven patients received sorafenib as a mono- or combined therapy. Three (43\%) of them showed SD after 3 months, and 4 (57\%) patients showed PD. Two of these 7 (29\%) patients had to stop the treatment due to adverse drug events (cutaneous toxicity). Three patients received ipilimumab and showed PD. One patient received bevacizumab combined with paclitaxel and carboplatin and showed SD after 3 months (table 3 ).

\section{Discussion}

\section{Local Therapy}

Our results highlight the difficult local tumor control in sinonasal melanoma with recurrence rates of up to 80 $90 \%$ in both $\mathrm{R} 0$ resected patients without additional radiotherapy as well as Rl resected patients with subsequent radiotherapy. Whether R0 resected patients do benefit from additional radiotherapy is discussed controversially $[3,4]$ and needs to be evaluated in a larger study population. Recent studies also showed that primary multilocular sinonasal melanoma does exist and forms a subtype that is associated with unfavorable local control independently of the postoperative procedure [5]. In contrast, local tumor control was better in vulvovaginal melanoma (7/8). However, in $4 / 6$ cases sentinel lymph node biopsy was positive and in $2 / 8$ cases radiological nodal involvement was detected at the time of first diagnosis. This suggests that diagnosis is established at a later time point compared to other melanomas. In uveal tumors, radiotherapy has been shown to be a legitimate alternative to radical surgery with similar long-term results for metastasization and overall survival $[6,7]$. The selection of the treatment procedure has to be evaluated for every patient individually and is based on uveal localization, tumor and patient characteristics [8-10]. In our cohort, local recurrence was lower for enucleation $(0 \% ; 0 / 22)$ than for radiotherapy $(25 \% ; 4 / 16)$. However, we did not distinguish between proton beam therapy, brachytherapy and radiotherapy, and interventions were spread over a large period of time. Other sources with larger populations reported much lower recurrence rates of between 1 and $6 \%$ after 5 years for proton beam therapy as well as for brachytherapy with a noticeable improvement over the last two decades $[8,11,12]$. In recent years, the tendency has therefore been towards favoring eye- and partial-visionconserving therapies. In conjunctival melanoma, the lesions arising de novo in contrast to arising from precursor ones along with local extension and corneal infiltration are the most predictive risk factors for tumor aggressiveness and systemic spreading probability. Complete surgical excision is the standard procedure and in cases of extensive involvement exenteration is necessary [13]. Local recurrence after conjunctival excision is common and makes further subsequent local treatment necessary [13-15].

\section{Tumor Mutations}

In the analyzed tumor samples, c-KIT mutation was found in vulvovaginal melanoma in 4 of 7 scanned cases $(57 \%)$ and was absent in sinonasal $(0 / 9)$ or ocular melanoma $(0 / 3)$. This is slightly higher than previously reported data that reported c-KIT mutations in approximately $20-40 \%$ of genital-mucosa melanomas and a much lower incidence in other mucosal melanomas [16-21]. BRAF mutation was not found in any of the 3 subgroups $(0 / 21$, i.e. $0 / 10$ sinonasal, $0 / 5$ vulvovaginal, $0 / 6$ ocular melano$\mathrm{ma}$ ), whereas NRAS mutation was detected in 1 sinonasal melanoma ( $1 / 7$, i.e. $1 / 4$ sinonasal, $0 / 2$ vulvovaginal, $0 / 1$ uveal) only. Zebary et al. [22] reported in a study on 56 cases similar low incidences of $4 \%$ for BRAF and c-KIT mutations and $14 \%$ for NRAS mutations in sinonasal melanomas. In ocular melanoma, the incidence of BRAF mutation varies strongly depending on the original tissue and localization. While BRAF mutation has been reported to reach a rate of up to $50 \%$ for iris melanoma [23] and 
about $22-29 \%$ for conjunctival melanoma $[24,25]$, it is often absent in the more prevalent posterior uveal melanoma (choroidal) [26, 27]. c-KIT mutation plays a minor role in ocular melanoma with low to absent incidences [28]. In contrast, GNA11 and GNAQ mutations are found regularly in uveal melanoma and have been shown to contribute to the development of malignancy by activating the MAPK pathway. Current trials are investigating the therapeutic effectiveness of MEK and PKC inhibitors [29]. Already routinely performed for uveal melanoma is the detection of chromosomal aberration (especially chromosome 3 loss and chromosome 8 gain) with high predictive value for prognosis in uveal melanoma and metastasization $[9,30,31]$.

\section{Localization of First Metastasis}

Sinonasal melanomas showed a heterogenic metastatic spread. Vulvovaginal melanoma on the other hand showed all first metastases in locoregional lymph nodes/ vessels. In contrast, first metastases in ocular melanomas were found in the liver in more than $80 \%$ (18/22) of cases, which corresponds to the described organotropy for uveal melanoma metastasization [32, 33]. However, combined/isolated metastases were found in the lungs (32\%) and bones (23\%) as well. Metastatic spread is therefore clearly different depending on primary tumor localization and has to be taken into account in tumor aftercare surveillance schemes. Damato et al. [10] developed a survival prediction tool for uveal melanoma based on multiple metastasization risk factors of the primary tumor to adapt aftercare and screening to individual risk.

\section{Systemic Treatment}

Immunotherapy

Overall 10 patients ( 4 sinonasal, 3 vulvovaginal, 3 ocular melanoma) received the anti-CTLA- 4 antibody ipilimumab $3 \mathrm{mg} / \mathrm{kg}$, whereof 1 patient (1/10) with sinonasal melanoma achieved tumor CR (10\%). Overall, tumor control was achieved in $3 / 10(30 \%)$ patients (including $\mathrm{CR}, \mathrm{PR}$ and $\mathrm{SD})$. One (1/10) patient suffered from a severe adverse drug reaction (colitis with sigma perforation, uveitis and otitis interna), and therapy had to be stopped early $(10 \%)$. These results correlate with the previously reported response rates for immunotherapy in mucosal melanomas of $3-12 \%$ PR and $16-43 \%$ SD as well as the published severe adverse drug reaction incidence $[34,35]$. Also, other authors described CR in single cases in mucosal and uveal melanoma $[35,36]$. For response to ipilimumab in uveal melanoma though, differing more modest data have been reported lately. While two previous studies provided similar data in 82 and 34 metastatic uveal melanoma patients treated with ipilimumab (PR rates of 2.6 and 5\%, SD rates of 29 and $43.4 \%$, respectively [36,37], and 1 CR [36]), no CR or PR was achieved in a recent phase II study including 53 patients [38]. Whether ipilimumab is considerably less effective in uveal melanoma compared to other noncutaneous melanomas needs to be investigated in further studies, but overall response seems to be similar as in cutaneous melanoma, and therefore ipilimumab should be considered a treatment in slow-growing noncutaneous melanoma.

In this patient population, only 1 patient (vulvovaginal melanoma) was treated with the anti-PD-1 antibody pembrolizumab after ipilimumab failure. This treatment resulted in an SD after 3 months. Recently very promising results with this new checkpoint inhibition have been reported in cutaneous melanoma $[39,40]$. It will be of great importance to consider anti-PD-1 antibodies in noncutaneous melanomas as well.

\section{Targeted Therapy}

c-KIT mutations were found as expected in vulvovaginal melanomas only $[19,21]$. All 3 mutated patients with systemic disease ( 2 mutated in exon $13 \mathrm{~K} 642 \mathrm{E}$ and 1 in exon $11 \mathrm{~L} 576 \mathrm{P}$ ) received the kinase inhibitor imatinib as systemic therapy. All 3 patients showed at least SD after 3 months. This result corresponds with the response rates to imatinib treatment described by Hodi et al. [41] with a PR rate of $53.8 \%$ and an SD rate of $23.1 \%$ for c-KIT-mutated melanomas $(n=13$, including 9 patients with c-KIT mutation in exon 11; other mutation sites included exon 17 and exon 13). As reported by Schoenewolf et al. [42], remarkable regression of lung, liver and bone metastases was observed under treatment with imatinib. However, brain metastases developed under the treatment. The lack of therapy efficacy for metastatic brain lesions despite good overall systemic response has been reported for imatinib-sensitive tumors arising from melanocytes as well as from other origins and may partially be explained by limited drug penetration into the central nervous system $[18,43,44]$. Development of secondary resistances has been documented for imatinib-treated melanoma patients as well (after 3.5-4.7 months) [45, 46]. Whether long-term efficacy could be improved by combining imatinib with other new targeted therapy agents needs to be evaluated in future studies.

Thirteen patients ( 6 sinonasal, 7 ocular melanoma) were treated with sorafenib monotreatment (10/13) or with a combined therapy $(3 / 13)$. The response rate was 
relatively low and did not necessarily correlate with treatment duration. In contrast, the frequency of severe adverse drug reaction was high and led to early therapy interruption in 6/13 of cases (46\%) due to the development of palmoplantar erythrodysesthesia, a common drugspecific reaction [47]. After 3 months of therapy, none of the patients showed PR, 4/13 (31\%) SD (whereof 2 had combined therapy with paclitaxel) and 9/13 (69\%) PD. These observations suggest that this agent might not be the optimal treatment choice for these patients. New promising data published by Carvajal et al. [48] reported an improved outcome in metastatic uveal melanoma when treated with the MEK inhibitor selumetinib. In our study, only 1 patient with uveal melanoma and unknown mutation status was treated within a clinical trial and showed PD after 3 months.

\section{Cytokines and Chemotherapy}

In our population 1 patient with vulvovaginal melanoma received adjuvant systemic subcutaneous interferon therapy for over 5 years and showed a particularly good course. Large studies have examined the positive effect of adjuvant immunotherapy with systemic interferons in cutaneous melanoma and reported the highest benefit for patients with ulcerated primary tumor and only marginal lymph node infiltration [49]. Since most of the mucosal melanomas are ulcerated and, especially in the vulvovaginal group, the majority of our patients showed microscopic lymph node infiltration, adjuvant interferon therapy might be beneficial. However, this must be evaluated in a larger cohort first.

Chemotherapy was applied to slow down progression when target or immunotherapy was ineffective and temporary $\mathrm{SD}$ was reached in some cases. As no curative approach can be taken with chemotherapy and the benefit on overall survival is marginal, it makes sense to choose potentially more effective therapies over chemotherapy until extended.

\section{Limitations}

This study has potential bias due to its retrospective setting and to its small sample size. Moreover, the study period is limited over time and might have failed to capture important disease course changes outside of this observation window. In addition, it is not possible for this analysis setting to draw conclusions on the singular concrete treatment effect on overall survival. Finally, systemic treatment of melanoma experienced big paradigm changes during the time period (2006-2013) of this study. The data of this study have therefore only a relative significance for today's treatment options.

\section{Conclusion}

Noncutaneous melanomas are rare, and this analysis gives a single-center overview of the different courses and treatments. Noncutaneous melanomas have a low presence of tumor-signaling pathway mutations and distinct metastasization patterns. Ocular melanomas are the most frequent noncutaneous melanomas and locally respond best to surgical and radiation treatment. Tumor mutations differ in conjunctival, iris and posterior uveal melanoma, and further exploration of the molecular biology should be performed to find vision-sparing targeted treatments. Imatinib is a good treatment option in the quite commonly c-KIT-mutated vulvovaginal melanomas, whereas treatment with sorafenib is highly associated with severe palmoplantar erythrodysesthesia and subsequent therapy interruption. Immunotherapy seems to be effective in noncutaneous melanomas with similar responses as in cutaneous melanomas and is therefore a legitimate therapy option in slow-growing disease. This suggests that anti-CTLA- 4 and more importantly antiPD-1 antibodies might hold promising responses for noncutaneous melanomas and should be evaluated in larger prospective studies.

\section{Statement of Ethics}

This single-center retrospective data analysis was approved by the local ethics committee (KEK-ZH No. 2014-0143).

\section{Disclosure Statement}

All authors (V.D.P., K.C., D.H., D.F., M.L., R.D. and S.M.G.) have no direct conflicts of interests to declare. R.D. receives research funding from Novartis, Bristol-Myers Squibb, Roche and GlaxoSmithKline, and has a consultant or advisory board relationship with Novartis, Merck Sharp \& Dohme, Roche, Bristol-Myers Squibb, GlaxoSmithKline and Amgen. S.M.G. has research funding from the University Hospital of Zurich and received travel grant support from MSD and BMS.
Del Prete/Chaloupka/Holzmann/Fink/ Levesque/Dummer/Goldinger 


\section{References}

1 McLaughlin CC, et al: Incidence of noncutaneous melanomas in the US. Cancer 2005; 103:1000-1007.

2 Bishop KD, Olszewski AJ: Epidemiology and survival outcomes of ocular and mucosal melanomas: a population-based analysis. Int J Cancer 2014;134:2961-2971.

3 Lund VJ, et al: Sinonasal malignant melanoma: an analysis of 115 cases assessing outcomes of surgery, postoperative radiotherapy and endoscopic resection. Rhinology 2012;50: 203-210.

4 Krengli $M$, et al: What is the role of radiotherapy in the treatment of mucosal melanoma of the head and neck? Crit Rev Oncol Hematol 2008;65:121-128.

5 Stanimirov Rossi O, et al: Multilocular sinonasal malignant melanoma: a poor prognostic subgroup? Eur Arch Otorhinolaryngol 2015; 272:123-129.

6 Puusaari I, et al: Iodine brachytherapy as an alternative to enucleation for large uveal melanomas. Ophthalmology 2003;110:22232234.

7 Hawkins BS: Collaborative ocular melanoma study randomized trial of I-125 brachytherapy. Clin Trials 2011;8:661-673.

8 Caujolle JP, et al: Local recurrence after uveal melanoma proton beam therapy: recurrence types and prognostic consequences. Int J Radiat Oncol Biol Phys 2013;85:1218-1224.

9 Prescher G, et al: Prognostic implications of monosomy 3 in uveal melanoma. Lancet 1996;347:1222-1225.

10 Damato B, et al: Estimating prognosis for survival after treatment of choroidal melanoma. Prog Retin Eye Res 2011;30:285-295.

11 Egger E, et al: Maximizing local tumor control and survival after proton beam radiotherapy of uveal melanoma. Int J Radiat Oncol Biol Phys 2001;51:138-147.

12 Damato B, et al: Local tumor control after ${ }^{106} \mathrm{Ru}$ brachytherapy of choroidal melanoma. Int J Radiat Oncol Biol Phys 2005;63:385-391.

13 Shields CL, et al: Conjunctival melanoma: outcomes based on tumor origin in 382 consecutive cases. Ophthalmology 2011;118: 389-395.

14 Shields CL, et al: Conjunctival melanoma: risk factors for recurrence, exenteration, metastasis, and death in 150 consecutive patients. Arch Ophthalmol 2000;118:1497-1507.

15 Damato B, Coupland SE: Management of conjunctival melanoma. Expert Rev Anticancer Ther 2009;9:1227-1239.

16 Curtin JA, et al: Somatic activation of KIT in distinct subtypes of melanoma. J Clin Oncol 2006;24:4340-4346.

17 Satzger I, et al: Analysis of c-KIT expression and KIT gene mutation in human mucosal melanomas. Br J Cancer 2008:99:2065-2069.
18 Handolias D, et al: Clinical responses observed with imatinib or sorafenib in melano ma patients expressing mutations in KIT. $\mathrm{Br}$ ] Cancer 2010;102:1219-1223.

19 Omholt K, et al: KIT pathway alterations in mucosal melanomas of the vulva and othe sites. Clin Cancer Res 2011;17:3933-3942.

20 Papaspyrou G, et al: Mucosal melanomas of the head and neck: new aspects of the clinical outcome, molecular pathology, and treatment with c-KIT inhibitors. Melanoma Res 2011;21:475-482.

21 Schoenewolf NL, et al: Sinonasal, genital and acrolentiginous melanomas show distinct characteristics of KIT expression and mutations. Eur J Cancer 2012;48:1842-1852.

22 Zebary A, et al: KIT, NRAS and BRAF mutations in sinonasal mucosal melanoma: a study of 56 cases. Br J Cancer 2013;109:559-564.

23 Henriquez $F$, et al: The T1799A BRAF mutation is present in iris melanoma. Invest Ophthalmol Vis Sci 2007;48:4897-4900.

$24 \mathrm{Gear} \mathrm{H}$, et al: BRAF mutations in conjunctival melanoma. Invest Ophthalmol Vis Sci 2004; 45:2484-2488

25 Griewank KG, et al: Conjunctival melanomas harbor BRAF and NRAS mutations and copy number changes similar to cutaneous and mucosal melanomas. Clin Cancer Res 2013; 19:3143-3152.

26 Spendlove HE, et al: BRAF mutations are detectable in conjunctival but not uveal melanomas. Melanoma Res 2004;14:449-452.

27 Cohen Y, et al: Lack of BRAF mutation in primary uveal melanoma. Invest Ophthalmo Vis Sci 2003;44:2876-2878.

28 Beadling $\mathrm{C}$, et al: KIT gene mutations and copy number in melanoma subtypes. Clin Cancer Res 2008; 14:6821-6828.

29 Sagoo MS, et al: Combined PKC and MEK in hibition for treating metastatic uveal melanoma. Oncogene 2014;33:4722-4723.

30 Shields CL, et al: Prognosis of uveal melano$\mathrm{ma}$ in 500 cases using genetic testing of fineneedle aspiration biopsy specimens. Ophthalmology 2011;118:396-401.

31 Damato B, et al: Cytogenetics of uveal melanoma: a 7-year clinical experience. Ophthalmology 2007;114:1925-1931.

32 Collaborative Ocular Melanoma Study Group: Assessment of metastatic disease status at death in 435 patients with large choroidal melanoma in the Collaborative Ocular Melanoma Study (COMS): COMS report No 15. Arch Ophthalmol 2001;119:670-676.

33 Damato BE, Coupland SE: Ocular melanoma. Saudi J Ophthalmol 2012;26:137-144.

34 Del Vecchio M, et al: Efficacy and safety of ipilimumab $3 \mathrm{mg} / \mathrm{kg}$ in patients with pretreated, metastatic, mucosal melanoma. Eur J Cancer 2014;50:121-127.

35 Postow MA, et al: Ipilimumab for patients with advanced mucosal melanoma. Oncologist 2013;18:726-732.
36 Luke IJ, et al: Clinical activity of ipilimumab for metastatic uveal melanoma: a retrospective review of the Dana-Farber Cancer Institute, Massachusetts General Hospital, Memorial Sloan-Kettering Cancer Center, and University Hospital of Lausanne experience. Cancer 2013;119:3687-3695.

37 Maio M, et al: Efficacy and safety of ipilimumab in patients with pre-treated, uveal melanoma. Ann Oncol 2013;24:2911-2915.

38 Zimmer L, et al: Phase II DeCOG study of ipilimumab in pretreated and treatment-naive patients with metastatic uveal melanoma. PLoS One 2015;10:e0118564.

39 Wolchok JD, et al: Nivolumab plus ipilimumab in advanced melanoma. N Engl J Med 2013;369:122-133.

40 Hamid O, et al: Safety and tumor responses with lambrolizumab (anti-PD-1) in melanoma. N Engl J Med 2013;369:134-144.

41 Hodi FS, et al: Imatinib for melanomas harboring mutationally activated or amplified KIT arising on mucosal, acral, and chronically sun-damaged skin. J Clin Oncol 2013;31: 3182-3190.

42 Schoenewolf NL, Urosevic-Maiwald M, Dummer R: Tumour heterogeneity of mucosal melanomas during treatment with imatinib. Br J Dermatol 2011;165:419-424.

43 Takayama N, et al: Imatinib mesylate has limited activity against the central nervous system involvement of Philadelphia chromosome-positive acute lymphoblastic leukaemia due to poor penetration into cerebrospinal fluid. Br J Haematol 2002;119:106-108.

44 Hughes B, et al: Cerebral relapse of metastatic gastrointestinal stromal tumor during treatment with imatinib mesylate: case report. BMC Cancer 2004;4:74.

45 Zukotynski $\mathrm{K}$, et al: Metabolic response by FDG-PET to imatinib correlates with exon 11 KIT mutation and predicts outcome in patients with mucosal melanoma. Cancer Imaging 2014; $14: 30$

46 Guo J, et al: Phase II, open-label, single-arm trial of imatinib mesylate in patients with metastatic melanoma harboring c-Kit mutation or amplification. J Clin Oncol 2011;29: 2904-2909.

47 Belloni B, et al: Cutaneous drug eruptions associated with the use of new oncological drugs. Chem Immunol Allergy 2012;97:191202.

48 Carvajal $\mathrm{RD}$, et al: Effect of selumetinib vs chemotherapy on progression-free survival in uveal melanoma: a randomized clinical trial. JAMA 2014;311:2397-2405.

49 Eggermont AM, et al: Ulceration and stage are predictive of interferon efficacy in melanoma: results of the phase III adjuvant trials EORTC 18952 and EORTC 18991. Eur J Cancer 2012; $48: 218-225$. 


\section{Curriculum Vitae}

Valerio Del Prete

25.02.1989

Geboren in Carmagnola (Italien)

1995-2001

Primarschule Männedorf

2001-2007

Kantonsschule Hohe Promenade in Zürich

Abschluss mit eidg. Matura (MAR), Profil Englisch/Latein

2007-2014

Humanmedizinstudium an der Universität Zürich

2014

2014-2015

Eidg.Staatsexamen in Humanmedizin an der Universität Zürich

seit 2015 Assistenzarzt Innere Medizin, Ärztehaus Bremgarten

Assistenzarzt Innere Medizin, Spital Zollikerberg 